\title{
Blowout bifurcation in a system of coupled chaotic lasers
}

\author{
Peter Ashwin and John R. Terry \\ Department of Mathematics and Statistics, University of Surrey, Guildford GU2 5XH, United Kingdom \\ K. Scott Thornburg, Jr. and Rajarshi Roy \\ School of Physics, Georgia Institute of Technology, Atlanta, Georgia 30332
}

(Received 18 February 1998)

\begin{abstract}
We show that loss of synchronization of two identical coupled chaotic class B lasers can occur via a blowout bifurcation. This occurs when a transverse Lyapunov exponent governing the stability of a synchronized subspace passes through zero. A system of two laterally coupled lasers with modulated parameters is investigated numerically in a region of chaotic behavior. A total of five invariant subspaces are shown to exist. Evidence of a blowout from one of these subspaces is found in Lyapunov exponents and in the presence of on-off intermittency for small enough coupling strengths. At all parameter values investigated, the phases of the electric fields are shown to be precisely synchronized even though the amplitudes may fluctuate chaotically and independently. We discuss the implication that there will be bubbling effects in laser systems in the presence of noise and imperfections. [S1063-651X(98)01012-5]
\end{abstract}

PACS number(s): 05.45.+b, 42.65.Sf, 42.55.Ah

Understanding the synchronization and desynchronization of signals from two or more nearly identical lasers is a matter that has important technological applications. Notably, it is important for designing high power coherent laser sources from arrays of low power lasers or for high-speed communication using synchronized optical systems. These applications have led to several studies of the problem of chaos and synchronization in lasers over the last few years [1-7].

We consider symmetric coupling of a pair of identical class B lasers [8]. In addition to the obvious symmetry of interchanging the lasers, there are phase-shift symmetries of the electric fields. An elementary but surprising consequence of these symmetries is the existence of states that are exactly phase-synchronized (synchronized) but not amplitude synchronized.

We observe a new route to loss of synchronization in laser systems. It is a symmetry breaking that is purely dynamical; i.e., caused by loss of stability of a synchronized attractor through a 'blowout' bifurcation, where a state exhibiting synchronization of both the phase and intensity loses stability to fluctuations that preserve only the phase synchronization. Such bifurcations have been seen numerically in maps [9-11] and experimentally in electronic and other systems [12-14] but this is, to our knowledge, the first observation of a blowout bifurcation in a laser system of any sort.

For physical systems, symmetry is only an idealization that is broken by imperfections in the system (e.g., where a perfectly synchronized state no longer exists [6]) or by noise within the system that moves the trajectory away from any synchronized state (e.g., [15]). In fact it is clear that only in exceptional cases will an exactly synchronized state be preserved on breaking the symmetry.

What we show is that such forced symmetry breaking is not necessary for desynchronization of lasers coupled by overlap of electric field; the mechanism we investigate leads to desynchronization without the need to appeal to either of these effects. Moreover, it is a form of desynchronization that will only occur when chaotic dynamics is present in the system.
The system we consider is a pair of coupled lasers where the coupling is purely via overlap of the electric field. The lasers under consideration are class B, where only the field and gain variables need be considered. The lasers are subjected to identical periodic modulations of the loss and may become chaotic in certain parameter regimes.

Our analysis is motivated by the two coupled single-mode class B lasers studied in Refs. [2,3,15]. We include a periodic forcing of the loss so that the two lasers are modulated at a rate close to the natural relaxation oscillation frequency; the frequency with which the laser intensity naturally fluctuates. Loss modulations at frequencies near the relaxation oscillation frequency have been shown to generate chaotic dynamics in similar systems $[16,17]$.

The lasers are assumed to be identical in all their parameters and they are subject to the same modulated loss; they are also assumed not to be detuned and thus frequency locked. Such lasers are governed by the following equations defining an evolution in the five dimensional phase space:

$$
\begin{gathered}
\frac{d X_{1}}{d T}=\left[F_{1}-\alpha_{0}\left(1+\alpha_{M} \cos \omega t\right)\right] X_{1}-\beta X_{2} \cos \Phi, \\
\frac{d F_{1}}{d T}=\gamma\left[A_{0}-F_{1}-F_{1} X_{1}^{2}\right], \\
\frac{d X_{2}}{d T}=\left[F_{2}-\alpha_{0}\left(1+\alpha_{M} \cos \omega t\right)\right] X_{2}-\beta X_{1} \cos \Phi, \\
\frac{d F_{2}}{d T}=\gamma\left[A_{0}-F_{2}-F_{2} X_{2}^{2}\right], \\
\frac{d \Phi}{d T}=\beta\left(X_{2} X_{1}^{-1}+X_{1} X_{2}^{-1}\right) \sin \Phi .
\end{gathered}
$$

$X_{i}$ represents the electric field amplitude, $F_{i}$ the gain of laser $i=1,2$, and $\Phi$ the difference in phases between the electric 
TABLE I. The symmetry-forced invariant subspaces of the equations for two coupled lasers. The first column gives a symbol for the subgroup of symmetries that fix a typical point in this invariant subspace with coordinates given by the second column $\left(X_{+}, F_{+}, X_{-}, F_{-}, \Psi\right.$ are arbitrary values for these coordinates). The third column gives the dimension of this invariant subspace within the five dimensional phase space.

\begin{tabular}{lccc}
\hline \hline Symmetry & Representative point & Dimension & Name \\
\hline $\mathbf{Z}_{2}(\kappa) \times \mathbf{Z}_{2}(\mu)^{+}$ & $\left(X_{+}, F_{+}, 0,0,0\right)$ & 2 & Synchronized \\
$\mathbf{Z}_{2}(\kappa) \times \mathbf{Z}_{2}(\mu)^{-}$ & $\left(X_{+}, F_{+}, 0,0, \pi\right)$ & 2 & Antisynchronized \\
$\mathbf{Z}_{2}(\mu)^{+}$ & $\left(X_{+}, F_{+}, X_{-}, F_{-}, 0\right)$ & 4 & Phase synchronized \\
$\mathbf{Z}_{2}(\mu)^{-}$ & $\left(X_{+}, F_{+}, X_{-}, F_{-}, \pi\right)$ & 4 & Phase antisynchronized \\
$\mathbf{Z}_{2}(\kappa \mu)$ & $\left(X_{+}, F_{+}, 0,0, \Psi\right)$ & 3 & Amplitude synchronized \\
\hline \hline
\end{tabular}

fields of the two lasers $\phi_{2}-\phi_{1}$. The equations are nondimensionalized with time being expressed in units of the round-trip time of light around the cavity, $\tau_{c} . A_{0}$ is the pump parameter, while the parameter $\gamma$ represents the ratio of the time scales of the electric field $\tau_{c}$ and the upper level spontaneous emission lifetime of the laser material $\tau_{f}$. We performed most of our simulations using $\gamma=10^{-2}$ to avoid stiffness problems that arise with smaller values of $\gamma$. For the Nd:YAG lasers (YAG denotes yttrium aluminum garnet) and the resonator configuration considered in Refs. [2,15] $\gamma \sim 10^{-6}$, but for other media (e.g., Ti: $\mathrm{AL}_{2} \mathrm{O}_{3}$ and $\mathrm{CO}_{2}$ [16]) or $\mathrm{Nd}: \mathrm{YAG}$ in longer resonators than those considered in $[2,3,15]$, we can obtain lasers with this more moderate value of $\gamma$.

The lasers are modulated with a depth $\alpha_{M}$ relative to their mean losses $\alpha_{0}$. In the absence of modulation both lasers are stable and exhibit damped oscillations to their fixed-point values. The coupling via $\beta$ is caused by the overlap of the laser electric fields in a laser crystal. If the beams have Gaussian profile with $1 / e^{2}$ radii $w_{0}$ and are separated by a distance $d$ then the coupling is proportional to the area of overlap between the two lasers, $\beta \sim e^{-d^{2} / w_{0}^{2}}[18]$.

Because we are interested in the problem of synchronization, we introduce the sum and difference variables, $X_{+}$ $=\frac{1}{2}\left(X_{1}+X_{2}\right), \quad X_{-}=\frac{1}{2}\left(X_{1}-X_{2}\right), \quad F_{+}=\frac{1}{2}\left(F_{1}+F_{2}\right), \quad F_{-}$ $=\frac{1}{2}\left(F_{1}-F_{2}\right)$, to facilitate the stability analysis of the synchronized state.

The transformed system is equivariant under the action of the symmetry

$$
\kappa\left(X_{+}, F_{+}, X_{-}, F_{-}, \Phi\right)=\left(X_{+}, F_{+},-X_{-},-F_{-},-\Phi\right)
$$

corresponding to interchanging the two lasers. There is another not so obvious symmetry of the system, namely

$$
\mu\left(X_{+}, F_{+}, X_{-}, F_{-}, \Phi\right)=\left(X_{+}, F_{+}, X_{-}, F_{-},-\Phi\right)
$$

as the only coupling is via $\cos (\Phi)$ terms. This corresponds to interchanging the phases of the beams without interchanging their amplitudes.

There is also a symmetry involving the parameter $\beta$; this adds $\pi$ onto $\Phi$ while reversing the sign of the parameter $\beta$. We use the parameter symmetry to simplify the numerics; however, this is not physically relevant as $\beta \geqslant 0$ in practice.

Because $\Phi$ is a periodic function, $\mu$ will fix the subspaces where $\Phi=0$ or $\pi$ and so there are in total five distinct dynamically invariant subspaces that are forced to exist purely by virtue of their symmetry. These are listed in Table I. Of particular interest are the existence of states we call phasesynchronized where the phases of the two lasers are identical and phase antisynchronized that are phase synchronized with a constant $\pi$ phase difference. Both of these phase synchronized states, surprisingly, do not show synchronization of their amplitude dynamics. Possibly even more surprising is the existence of states we denote as amplitude synchronized where the amplitudes are identical but the phases are not. However, these last states are not observed to be attractors of the system in the absence of detuning.

For $0<\gamma \ll 1$ the system undergoes a period doubling cascade to chaos as the strength of modulation $\alpha_{M}$ is increased, for values of the forcing frequency close to the relaxation oscillation frequency (cf. [16]). Any attractor is contained either in the phase-synchronized or phaseantisynchronized subspaces. This is because for any $\left(X_{1}, X_{2}\right)$ bounded away from zero we have $d \Phi / d t$ $=\beta F \sin \Phi$ with $F$ positive and bounded below. Therefore $\Phi \rightarrow \pi$ as $t \rightarrow \infty$ for almost any initial condition, and any attractor must be contained within the phaseantisynchronized subspace. Note that if $\beta<0$ then the above holds but with the phase-synchronized and phaseantisynchronized subspaces exchanged.

The simulations were performed using Bulirsch Stoer and Runge-Kutta integrators. We consider here only the case of modulated loss but note that we have found similar results for modulated pumping. For a typical value of $\gamma=0.01$ and $A_{0}=1.2, \alpha_{0}=0.9$ and $\alpha_{M}=2 / 9$ we see, on varying the coupling strength $\beta$, that there is a critical value of $\beta, \beta_{c}$ $\sim 0.002234$, such that a randomly chosen initial condition evolves as follows. For $0<\beta<\beta_{c}$ the trajectory is attracted onto the phase-antisynchronized subspace to a chaotic attractor that intersects but is not contained within the antisynchronized subspace. For $\beta>\beta_{c}$ there is an attractor within the antisynchronized subspace. As explained above, the phase difference $\Phi$ always evolves to $\pi$.

Figures 1(a) and 1(b) demonstrate the amplitude dynamics [given in terms of the sum $\left(X_{+}\right)$and difference $\left(X_{-}\right)$ variables] for a fully synchronized attractor with $\beta>\beta_{c}$. For contrast, Figs. 1(c) and 1(d) show an attractor with occasional large fluctuations away from the synchronized subspace reminiscent of on-off intermittent behavior at $\beta<\beta_{c}$. The transition at $\beta_{c}$ is strongly suggestive of a blowout bifurcation [9].

To investigate the loss of synchronization at $\beta=\beta_{c}$ and confirm the blowout scenario we numerically compute the Lyapunov exponents of attractors by integrating the varia- 

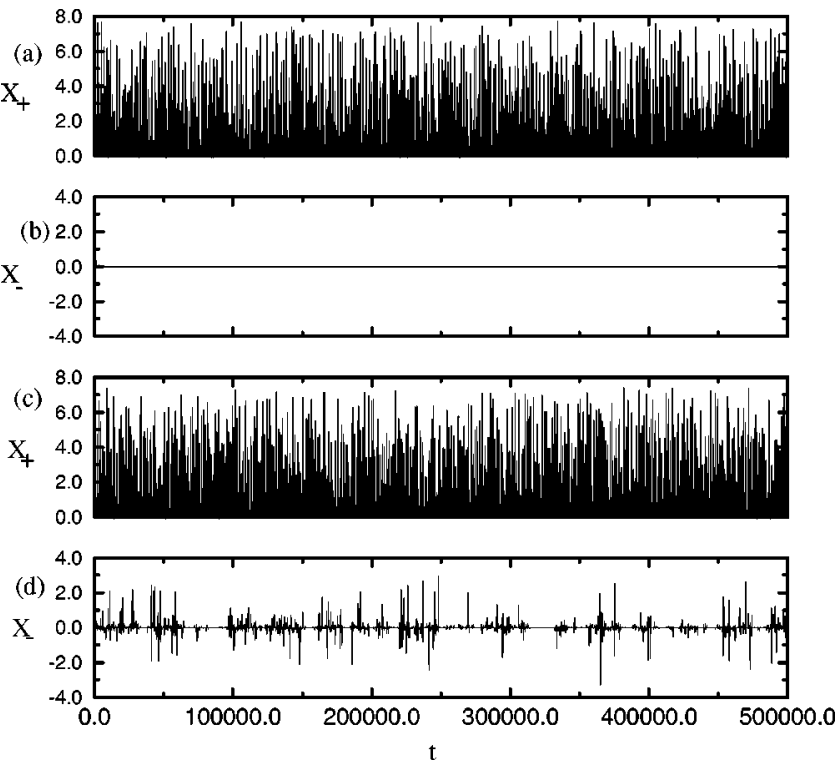

FIG. 1. Numerically calculated electric field amplitudes in a loss-modulated laser, computed by integrating Eqs. (1) with randomly chosen initial conditions. $X$ is shown in arbitrary units, $t$ in units of the round-trip lifetime of the laser. Figures (a) and (b) show the intensity sum $\left(X_{+}\right)$and difference $\left(X_{-}\right)$variables, respectively, at a coupling $\beta=0.003$, and the complete synchronization of their intensities typical of dynamics on the (anti)synchronized attractor. (c) and (d) show the intensity sum and difference variables at a value of $\beta=0.002$ that is less than $\beta_{c}$. On-off intermittent behavior is seen in the occasional, large fluctuations away from the (anti)synchronized attractor.

tional equations; recall that a blowout bifurcation occurs when the largest transverse Lyapunov exponent of the attractor for the system within an invariant subspace passes through zero. This Lyapunov exponent governs the exponential rate of growth of almost all perturbations away from the invariant subspace and in particular when it is negative the attractor within the subspace is the attractor for the full system.

Suppose we have a trajectory $\left(x_{+}(t), f_{+}(t), 0,0, \pi\right)$ for an initial condition chosen randomly for the system (1) in the subspace $\mathbf{Z}_{2}(\kappa) \times \mathbf{Z}_{2}(\mu)^{-}$(the antisynchronized subspace) and consider the behavior of a point $\left(x_{+}(t)+\delta x_{+}, f_{+}(t)\right.$ $\left.+\delta f_{+}, \delta x_{-}, \delta f_{-}, \pi+\delta \phi\right)$ linearized about the $\delta$ variables. The $\delta$ terms represent small perturbations away from the trajectory. Perturbations with $\delta x_{-}=\delta f_{-}=\delta \phi=0$ correspond to perturbations within the antisynchronized subspace and these grow at a rate $e^{\Lambda t}$ where $\Lambda$ is some tangential Lyapunov exponent $\Lambda_{1}$ or $\Lambda_{2}$. Any other perturbation will grow at a rate $e^{\lambda t}$ where $\lambda$ is a transverse Lyapunov exponent. If any of these transverse Lyapunov exponents are positive, the antisynchronized subspace is unstable. Since the antisynchronized subspace is codimension 3, there are three transverse Lyapunov exponents. We can divide these up into a pair, $\lambda_{1}$ and $\lambda_{2}$, corresponding to perturbations within the phase-antisynchronized subspace and one $\lambda_{3}$ that breaks phase locking. It is easy to compute from the linearization of the last equation of Eq. (1) that $\lambda_{3}=-2 \beta$.

To see precisely when the antisynchronized state is attracting, we have numerically computed $\lambda_{i}<0$ for $i=1,2$. Figure 2 shows a scan through a range of $\beta$ showing the

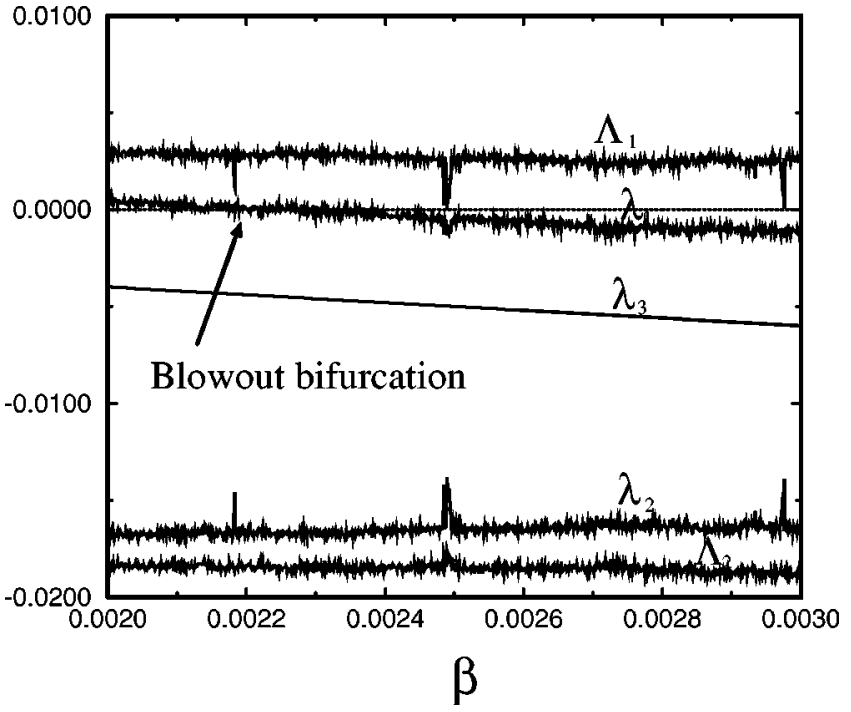

FIG. 2. Scan through parameter space showing the variation of the Lyapunov exponents with $\beta$ for an initial condition started within the antisynchronized subspace, parameter values as in Fig. 1. The $\Lambda_{i}$ are tangential Lyapunov exponents whereas the $\lambda_{i}$ are transverse Lyapunov exponents. N.B., (a) The most positive $\lambda_{1}$ passes through 0 at $\beta \sim 0.00223$ indicating a blowout bifurcation occurs here, (b) $\Lambda_{1}$ is positive, indicating the presence of chaotic behavior, (c) there are occasional dips in $\Lambda_{1}$ corresponding to windows of stabilization of periodic attractors in the antisynchronized subspace, (d) $\lambda_{3}=-2 \beta$ exactly.

tangential and transverse Lyapunov exponents. This was computed using trajectories with length 150000 and orthonormalization of the variational equations. By making a linear fit on the obtained values of $\lambda_{1}$ we compute that the blowout occurs at approximately $\beta_{c}=0.002234$. For $\beta<\beta_{c}$ there is an attracting on-off intermittent state that persists up to the point of the blowout bifurcation. We have also investigated cases for $\gamma=10^{-3}$ and $5 \times 10^{-4}$ and observed similar behavior, with the additional observation that the blowout bifurcations occur for progressively smaller values of $\beta$ as the stiffness parameter $\gamma$ is reduced. Due to the increased stiffness of the equations, the numerics become much harder to evaluate accurately in this limit, and machine precision becomes an important issue when computing the normal Lyapunov exponents.

In conclusion, we demonstrate there can be a blowout bifurcation in a system of two coupled lasers with periodically modulated parameters. This is a dynamical symmetry breaking within the system that leads to a desynchronization of the amplitudes of the two chaotic lasers, comparable to bifurcations studied in $[9,11,12]$. The desynchronized attractors are still exactly phase (anti)synchronized due to other symmetries of the system.

In the experiment of [2], desynchronizing of two nearidentical Nd:YAG lasers was observed to occur on increasing the distance between them and hence decreasing the coupling; this fits well to our numerical observations even though in the experiment (a) pump modulation was used instead of loss modulation and (b) the values of $\gamma$ were of the order of $10^{-6}$. The similarity in the qualitative behaviors lead us to believe that the blowout is an important mechanism for loss of stabilty in the experiment even though it will 
be modified (as detailed below) in the presence of noise and imperfections.

For real systems such as in [2], noise and imperfections in the symmetry are both unavoidable and these will play a decisive role in determining the dynamics. Noise and symmetry breaking have similar effects; in the regime of on-off intermittency, very little change will be noticeable if perturbations are small. Before the blowout, however, the presence of bubbling [12] will tend to create on-off intermittentlike dynamics that will persist up to a bubbling transition [23]. Thus, in the presence of imperfections, the blowout scenario should still be present with the following modifications. On decreasing the coupling strength there should be a transition to bubbling; this will change smoothly into an on-off intermittency regime. If there is a mismatch in the parameters of the lasers, for example, if there is detuning, then this will destroy some of the invariant subspaces and cause the phase dynamics to become more nontrivial, as discussed, for example, in [15]. This will presumably also result in bubbling effects. The effects of on-off intermittency and bubbling have also recently been observed in simulations of coupled master-slave laser systems by Sauer and Kaiser [19].

One consequence of this investigation is that we expect the coupling strength for synchronizing such linearly coupled chaotic lasers will be intimately related to the magnitude of the positive Lyapunov exponent of the synchronized chaos, as discussed by Schuster et al. [20]. In particular, if the (anti)synchronized state is nonchaotic, e.g., attracting periodic, then it will be stable and no blowout will be in evidence. As is evident in the "coarseness" of the graph of Lyapunov exponents against $\beta$ in Fig. 2, the fact that $\beta$ is not a normal parameter [21] (i.e., $\beta$ varies the dynamics within the synchronized subspace as well as that transverse to it) means that we do not expect these exponents to vary smoothly or even continuously; see [22].

P.A. and J.T. would like to thank the EPSRC for their support via Grant No. GR/K77365. R.R. and K.S.T.J. acknowledge the support from the U.S. Office of Naval Research.
[1] H. G. Winful and L. Rahman, Phys. Rev. Lett. 65, 1575 (1990).

[2] R. Roy and K. S. Thornburg, Jr., Phys. Rev. Lett. 72, 2009 (1994).

[3] P. Colet and R. Roy, Opt. Lett. 19, 2056 (1994).

[4] T. Sugawara, M. Tachikawa, T. Tsukamoto, and T. Shimizu, Phys. Rev. Lett. 72, 3502 (1994).

[5] Y. D. Liu, P. C. de Oliveira, M. B. Danailov, and J. R. Rios Leite, Phys. Rev. A 50, 3464 (1994).

[6] Y. Liu and J. R. Rios Leite, Phys. Lett. A 191, 134 (1994).

[7] G. D. VanWiggeren and R. Roy, Science 279, 1198 (1998).

[8] F. T. Arecchi, G. L. Lippi, G. P. Puccioni, and J. R. Tredicce, Opt. Commun. 51, 308 (1994).

[9] E. Ott and J. C. Sommerer, Phys. Lett. A 188, 39 (1994).

[10] H. Fujisaka and T. Yamada, Prog. Theor. Phys. 69, 32 (1983).

[11] A. S. Pikovsky and P. Grassberger, J. Phys. A 24, 4587 (1991).

[12] P. Ashwin, J. Buescu, and I. Stewart, Phys. Lett. A 193, 126 (1994).

[13] L. M. Pecora, T. L. Carroll, and J. F. Heagy, Proc. SPIE 2612, 25 (1995).
[14] A. Cenys, A. Namajunas, A. Tamasevicius, and T. Schneider, Phys. Lett. A 213, 259 (1996).

[15] K. S. Thornburg, Jr., M. Möller, R. Roy, T. W. Carr, R.-D. Li, and T. Erneux, Phys. Rev. E 55, 3865 (1997).

[16] D. Dangoisse, P. Glorieux, and D. Hennequin, Phys. Rev. A 36, 4775 (1987).

[17] J. R. Tredicce, N. B. Abraham, G. P. Puccioni, and F. T. Arecchi, Opt. Commun. 55, 131 (1985).

[18] L. Fabiny, P. Colet, R. Roy, and D. Lenstra, Phys. Rev. A 47, 4287 (1993).

[19] M. Sauer and F. Kaiser, Phys. Lett. A 243, 38 (1998).

[20] H. Schuster, S. Martin, and W. Martienssen, Phys. Rev. A 33, 3547 (1986).

[21] P. Ashwin, J. Buescu, and I. N. Stewart, Nonlinearity 9, 703 (1996).

[22] P. Ashwin, E. Covas, and R. Tavakol, University of Surrey Department of Mathematics and Statistics Technical Report No. 98/3, 1998.

[23] S. C. Venkataramani, B. R. Hunt, E. Ott, D. J. Gauthier, and J. C. Bienfang, Phys. Rev. Lett. 77, 5361 (1996). 